\title{
Obituary: Predhiman Krishan Kaw
}

\author{
Abhijit Sen ${ }^{1}$
}

Published online: 10 October 2017

(c) Division of Plasma Physics, Association of Asia Pacific Physical Societies 2017

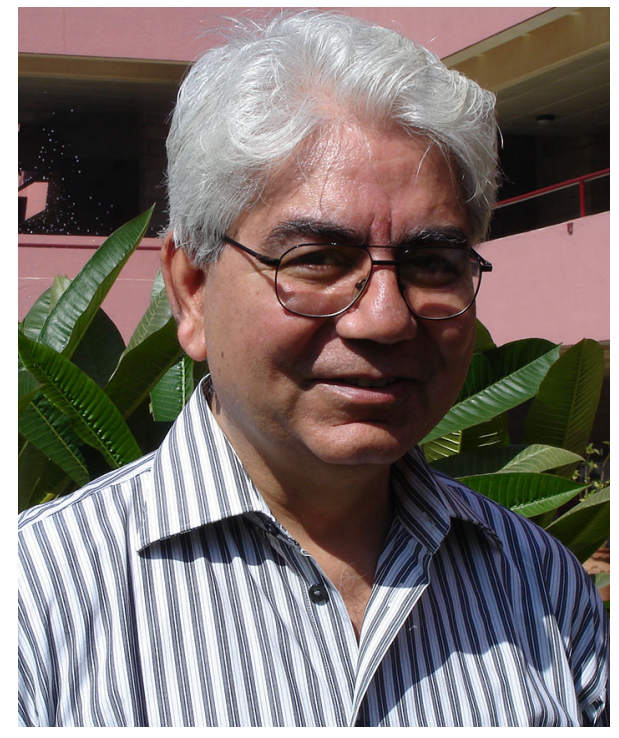

Prof. Predhiman Krishan Kaw, an internationally acclaimed plasma physicist and the chief architect of India's nuclear fusion program passed away suddenly on 18th June, 2017. A brilliant scientist who made significant contributions to many areas of plasma physics, he was also a wonderful teacher, a prolific researcher (with over 400 research publications), an inspiring leader and a man of great scientific vision.

\section{Abhijit Sen}

senabhijit@gmail.com

1 Institute for Plasma Research, Near Indira Bridge, Bhat, Gandhinagar 382428, India 
Predhiman was born in Kashmir on 15 January 1948, and was a child prodigy who received his early schooling at home and went on to obtain his Master's degree at the age of 16 from the MMH College in Ghaziabad. He then joined the Indian Institute of Technology, Delhi and was awarded a Ph.D. degree at the age of 18 . Thereafter, he joined the Princeton Plasma Physics Laboratory for postdoctoral research. His work during this period of 1967-1971 that resulted in seminal contributions to nonlinear physics of laser-plasma interactions laid the foundations for much of the current research on high power laser matter interactions. Among his notable contributions are, laser-induced parametric instabilities which lead to anomalously large absorption as well as back and side-scattering, filamentation of laser light due to ponderomotive forces in a plasma and relativistic nonlinear effects which have also found application in pulsar radiation phenomena. From 1971 to 1975, he worked at the Physical Research Laboratory (PRL), Ahmedabad, India, where he made important contributions to the theory of ionospheric irregularities. He also initiated laboratory experiments to simulate some of the ionospheric phenomena thereby planting a seed for future experimental plasma physics activities in India.

In 1975, Predhiman went back to Princeton and took up research on magnetically confined fusion plasmas and made several pioneering contributions in this area. He showed that the decades old conventional wisdom on the stability of drift waves in sheared geometry (a prime candidate for transport in fusion devices) was incorrect. He also demonstrated the existence of a coalescence instability of magnetic islands that can be important in diverse phenomena like disruption in tokamaks, energy release in solar flares and sub-storm effects in tail regions of the magnetosphere.

While still in the US, he noticed the remarkable and rapid progress of tokamak research at the international level and felt that the time was right for India to invest its intellectual and material resources towards the development of this future source of energy. Accordingly, he conceived and spearheaded the effort to establish a magnetic fusion program in India. In the late 70s and early 80 s, he and some of his colleagues at PRL succeeded in persuading the Department of Science and Technology, Government of India, to set up a major program of plasma physics at PRL. He returned to India in 1982 to head this program, which eventually evolved into the Institute for Plasma Research. Under his leadership and guidance, the Institute made remarkable progress on several fronts: (1) A medium-sized tokamak device called ADITYA was indigenously designed and fabricated. This machine was commissioned in 1989 and has led to some very novel results on intermittency in tokamak edge turbulence. (2) A large number of basic experiments investigating fundamental processes in plasmas were set up and have led to exciting new physics. (3) The Institute also successfully developed a number of plasma processing technologies and some of these technologies have been transferred to Indian industries. (4) The Institute carried out the design/fabrication of an advanced steadystate superconducting tokamak SST-1. This machine was commissioned in 2014. (5) The Institute successfully spearheaded India's case for participation in the prestigious ITER experiment and is now the nodal domestic agency managing this participation. During the formative years of ITER, Predhiman also played a very important role as Chairman of the ITER STAC, the Science and Technology 
Advisory Committee of ITER, by making sure that there was no compromise in the final scientific and technical objectives of ITER. (6) The Institute nurtured and trained a large number of young plasma physicists and technical personnel through its graduate program and technical training programs to cater to the rapidly growing R\&D activities in the field. Thus, in the span of a few years he was successful in setting up a thriving research and technology development program in the country and to put India on the world map of fusion research.

Despite his hectic schedule and commitments towards the development of such a large research and technology program, he never compromised on his personal research work and continued to actively pursue it. In a pioneering effort, he and his colleagues discovered the phenomenon of intermittency in tokamak edge plasma turbulence of ADITYA - a result that spawned a lot of further research in other tokamaks of the world. At this stage Predhiman also enlarged his research interests to more fundamental areas like dynamics of quark-gluon plasmas, equilibria of nonneutral electron clouds and collective effects in dusty plasmas and other strongly coupled systems. In each of these areas he provided new ideas, valuable insights and in many cases strong incentives and frameworks for laboratory experiments. He also continued his investigations on nonlinear phenomena in relativistic light-plasma interactions and made important contributions to the theory of envelope solitons and to the physics of anomalous stopping of fast electrons-topics of great interest for laser plasma-based particle acceleration schemes and the fast ignition scheme of laser fusion. He extended activities in these frontier areas of science and technology by forging collaborations with other active research groups within the country and abroad. One fine example is the establishment of collaboration between the theory and simulation activities at IPR with TIFR experiments in the field of laser-plasma interactions. This collaboration has led to fundamental insights in issues pertaining to laser energy absorption, magnetic field generation, its evolution and its turbulent characterization in laboratory laser-plasma experiments. These ideas are tied up closely with the physics of fast ignition in laser fusion and have also been found relevant to the experiments on Laser Fusion in Osaka.

For his outstanding contributions and achievements, Predhiman received many honors and awards including the Indian National Science Academy's Young Scientist Award in 1974, the Padma Shri award in 1985, the SS Bhatnagar award in 1986, the IPA R.D. Birla Award in 2002 and the TWAS Prize in Physics in 2008. Most recently, he was awarded the Subrahmanyan Chandrasekhar Prize for "outstanding contributions" in the field of plasma physics by the Association of Asia Pacific Plasma Physics Societies. He was a Fellow of all the major science academies in India and also the American Physical Society.

Predhiman was a passionate scientist and a great teacher who never tired of promoting the cause of science and fusion. His Artsimovich Memorial Lecture delivered in the opening session of the IAEA Fusion Energy Conference at Wurzburg, Germany in 1992 made a lasting impression on the international community and was a stunning example of his remarkable perspective on the state of international fusion research at the time and his clear vision on the path forward. He was a great believer and a champion of fusion research to whom the international community frequently looked up for guidance and inspiration. To 
prepare the next generation for taking this task forward he also spent an enormous time mentoring students and younger colleagues. He strongly believed that science should be pursued with complete freedom of thought and implementation. When the IPR buildings were planned, he engaged the architects and some of his colleagues in endless discussions to help incorporate this concept into the design. The wide corridors, the openness to the sky and the surroundings, the oneness with nature and the sense of liberation that they bring are all embodiments of this conviction. Likewise, his office door was always open for anybody to step in and to discuss science with him. As a human being he was extra-ordinarily kind and gentle with infinite patience particularly for younger colleagues. This coupled with his infectious enthusiasm for research, his sense of humor and informal behavior attracted many students and scientists, not only from within the Institute, but also from the Universities and the scientific community abroad as research collaborators. Each one of them felt enriched and emotionally touched by his warmth and generosity of spirit. In his more than 50 years of active scientific life, Predhiman has not only contributed significantly to the progress of plasma physics worldwide but has also inspired a lot of young minds and touched a great many human lives in an uplifting manner. He will be greatly missed by all of them. He leaves behind an invaluable legacy in science and humanity.

Prof. Kaw is survived by his wife Saroj and children, Sidharth, Prashant and Puja. - Abhijit Sen 